OPEN ACCESS

Edited by:

Yujing $L i$,

Emory University, United States

Reviewed by:

Melanie Carless,

Texas Biomedical Research Institute,

United States

Andrew Shafik,

Emory University, United States

Xingshun Xu,

The Second Affiliated Hospital

of Soochow University, China

*Correspondence:

Lu Shen

shenlu@csu.edu.cn

shenlu2505@126.com

Specialty section:

This article was submitted to

$R N A$,

a section of the journal

Frontiers in Genetics

Received: 04 June 2018 Accepted: 08 November 2018 Published: 30 November 2018

Citation:

Liu X, Jiao B and Shen L (2018)

The Epigenetics of Alzheimer's

Disease: Factors and Therapeutic Implications. Front. Genet. 9:579.

doi: 10.3389/fgene.2018.00579

\section{The Epigenetics of Alzheimer's Disease: Factors and Therapeutic Implications}

\author{
Xiaolei Liu'1,2, Bin Jiao ${ }^{1,3,4}$ and Lu Shen 1,3,4,5* \\ ${ }^{1}$ Department of Neurology, Xiangya Hospital, Central South University, Changsha, China, ${ }^{2}$ The Center of Gerontology \\ and Geriatrics, West China Hospital, Sichuan University, Chengdu, China, ${ }^{3}$ National Clinical Research Center for Geriatric \\ Disorders, Central South University, Changsha, China, ${ }^{4}$ Key Laboratory of Hunan Province in Neurodegenerative Disorders, \\ Central South University, Changsha, China, ${ }^{5}$ Key Laboratory of Organ Injury, Aging and Regenerative Medicine of Hunan \\ Province, Changsha, China
}

Alzheimer's disease $(A D)$ is a well-known neurodegenerative disorder that imposes a great burden on the world. The mechanisms of $A D$ are not yet fully understood. Current insight into the role of epigenetics in the mechanism of AD focuses on DNA methylation, remodeling of chromatin, histone modifications and non-coding RNA regulation. This review summarizes the current state of knowledge regarding the role of epigenetics in $\mathrm{AD}$ and the possibilities for epigenetically based therapeutics. The general conclusion is that epigenetic mechanisms play a variety of crucial roles in the development of $A D$, and there are a number of viable possibilities for treatments based on modulating these effects, but significant advances in knowledge and technology will be needed to move these treatments from the bench to the bedside.

Keywords: Alzheimer's disease (AD), epigenetics, non-coding RNA (ncRNA), DNA methylation, histone modifications and chromatin structure

\section{INTRODUCTION}

Alzheimer's disease (AD), an irreversible and progressive neurological disease, is the most common form of neurodegenerative dementia (Mckhann et al., 2011; Rocca et al., 2011; Lansdall et al., 2017). The most prominent early symptom is short-term memory loss. As the disease progresses, other symptoms such as personality changes, apathy and language problems emerge. 95 percent of patients who are hospitalized with $\mathrm{AD}$ have the sporadic form, which is late-onset Alzheimer's disease (LOAD) (Diniz et al., 2017; Zhao et al., 2017). The pathology of LOAD is multi-factorial with biological, genetic and environmental factors interacting with each other to aggravate the process of AD. Many genetic risk factors for LOAD have been identified. Among them, the $\varepsilon 4$ isoform of apolipoprotein $\mathrm{E}$ (ApoE4) is well-known as the strongest genetic risk factor for LOAD (Lane-Donovan and Herz, 2017). However, less than 1 percent of patients have autosomal dominant inherited Alzheimer's disease (DIAD) which has a much earlier age of onset, around 45 years old. Genetic mutations in the genes encoding APP, PS1, and PS2 that cause overproduction or formation of an aberrant form of $A \beta$ are found in this group (Levy et al., 1990; Masters et al., 2015).

At the cellular level, $\mathrm{AD}$ can be characterized by the appearance of extracellular plaques from accumulations of insoluble amyloid beta $(\mathrm{A} \beta)$ filaments, intracellular neurofibrillary tangles of hyperphosphorylated tau and neuroinflammation (Selkoe, 2012). It appears that AD is not only 
one or two types of diseases, but is rather a group of diseases with similar APP and Tau pathologies that are triggered by different mechanisms (Sery et al., 2013). Among them, the most popular theory is the amyloid cascade hypothesis and detection of the accumulation of $\mathrm{A} \beta$ is now available in MCI and $\mathrm{AD}$ patients using cerebrospinal fluid biomarkers and PET. However, it is still difficult to find stable biomarkers which could help to discover this disease much earlier, and the exact mechanisms of $\mathrm{AD}$ are still unknown. Now the number of studies examining epigenetic mechanisms playing in the etiology of $\mathrm{AD}$ has risen dramatically. With this increased focus, the epigenetic modification in $\mathrm{AD}$ has become a highly popular topic. This review summarizes the literature on the role of epigenetic mechanisms in the development of $\mathrm{AD}$ and the possibilities for treating $\mathrm{AD}$ by epigenetically based interventions.

\section{EPIGENETICS FACTORS IN AD}

Epigenetic factors include DNA methylation, histone modifications, chromatin remodeling, and regulation by non-coding RNA (Figure 1) (Morange, 2002; Holliday, 2006; Santana et al., 2017). The functions of epigenetics have been studied in various areas of biology, cancer biology and so on (Jones and Laird, 1999; Champagne, 2013). Now, many researchers are focusing on investigating the potential roles of epigenetics in $\mathrm{AD}$ pathogenesis.

\section{DNA METHYLATION}

DNA methylation modifies cytosine residues by adding methyl groups in cytosine/guanine-rich regions such as $\mathrm{CpG}$ islands (Mehler, 2008). The process is initiated by DNA methyltransferases (DNMT), including DNMT1, DNMT2, DNMT3a, and DNMT3b (Kemme et al., 2017). In this section we discuss the possibility that $\mathrm{AD}$ could be caused by aberrations in DNA methylation in some certain genes and the potential role of methylation in being a biomarker in AD.

For example, it was found that some cytosines, particularly those at -207 to approximately -182 , in the promoter region of the APP gene are mostly methylated and that their demethylation with age may led to $A \beta$ deposition in the aged brain (Tohgi et al., 1999a,b). Methylation of the microtubule-related protein tau (MAPT) gene could also suppress the MAPT expression, which could affect the levels of tau protein (Zhang et al., 2016). Mano et al. (2017) used postmortem brain samples from $\mathrm{AD}$ patients and found that the expression of BRCA1 was significantly upregulated, consisting of its hypomethylation. Additionally, BRCA1 protein levels were increased in response to $\mathrm{A} \beta$ and became mislocalized to the cytoplasm, in both in vitro cellular and in vivo mouse models (Mano et al., 2017). Recently, it was found that reduced DNA methylation at the triggering receptor expressed on myeloid cells 2 (TREM2) gene intron 1 caused higher TREM2 mRNA expression in the leukocytes of AD subjects than in the controls (Ozaki et al., 2017). Furthermore, DNA methylation in some other genes also plays a role in the mechanisms of $\mathrm{AD}$ and might be a potential biomarker. A study found that DNA methylation (CpG5) of the BDNF gene promoter and a tag SNP (rs6265) have a significant role in the etiology of amnestic mild cognitive impairment (aMCI) and its progression to $\mathrm{AD}$ (Xie et al., 2017a). In addition, a 5-year longitudinal study using multivariate Cox regression analysis revealed that elevated methylation of CpG5 of BDNF promoters was a significant independent predictor of $\mathrm{AD}$ conversion. This suggests that increased levels of peripheral BDNF promoter methylation may be an epigenetic biomarker indicating the transformation of aMCI to AD (Xie et al., 2017b). Kobayashi et al. (2016) examined the DNA methylation levels of the COASY and SPINT gene promoter regions and found that DNA methylation in the two regions was significantly increased in $\mathrm{AD}$ and aMCI as compared to controls. Di Francesco et al. (2015) found that global DNA methylation in the peripheral blood mononuclear cells of LOAD patients was higher compared to healthy controls, and higher DNA methylation levels were associated with the presence of APOE $\varepsilon 4$ allele $(p=0.0043)$ and $\mathrm{APOE} \& 3$ carriers $(p=0.05)$ in the global population. This indicated global DNA methylation in peripheral samples is a useful marker for screening individuals at risk of developing AD (Di Francesco et al., 2015). Moreover, DNA methylation levels in the NCAPH2/LMF2 promoter region were found to be a useful biomarker for the diagnosis of AD and aMCI (Shinagawa et al., 2016). One study has found that APOE CGI holds lower DNA methylation levels in $\mathrm{AD}$ compared to control in the frontal lobe, mostly in the non-neuronal cells of the AD brain (Tulloch et al., 2018). Additionally, PICALM gene methylation was found to be associated with cognitive decline in blood cells of AD patients (Mercorio et al., 2018). Recently, it was discovered that elevated DNA methylation across a 48-kb region spanning the HOXA gene cluster is associated with AD neuropathology (Smith et al., 2018). Moreover, a differentially methylated region was identified within the alternative promoter of the PLD3 gene showing higher DNA methylation levels in the AD hippocampus compared to controls (Blanco-Luquin et al., 2018). All of these indicated that the methylation levels of some genes could be potential biomarkers in AD.

In some postmortem brains or neuronal cells of $\mathrm{AD}$ individuals, a lot of work has been done to find more specific evidence. In cortical neurons of a postmortem $\mathrm{AD}$ brain, immunoreactivity for 5-methylcytosine (5-mC) was decreased compared to the control (Mastroeni et al., 2010). Levels of $5 \mathrm{mC}$ were reduced in the hippocampus, entorhinal cortex and cerebellum of patients with AD (Chouliaras et al., 2013; Condliffe et al., 2014). In hippocampal tissue, Mastroeni et al. (2010) found weak staining for antibodies against DNA methylation maintenance factors in $\mathrm{AD}$ cases, contrasting with normal brains. More compellingly, in a comparison of cortical neurons of monozygotic twins, one of whom had $\mathrm{AD}$ and the other not, they found extensive co-localization of $5 \mathrm{mC}$, with three cell-specific markers in the non-AD twin yet an absence of co-localization in the AD twin (Mastroeni et al., 2009). Furthermore, a study identified that numerous $\mathrm{AD}$-related genes, such as MCF2L, ANK1, MAP2, LRRC8B, STK32C, and S100B, have cell-type-specific methylation signatures and document 


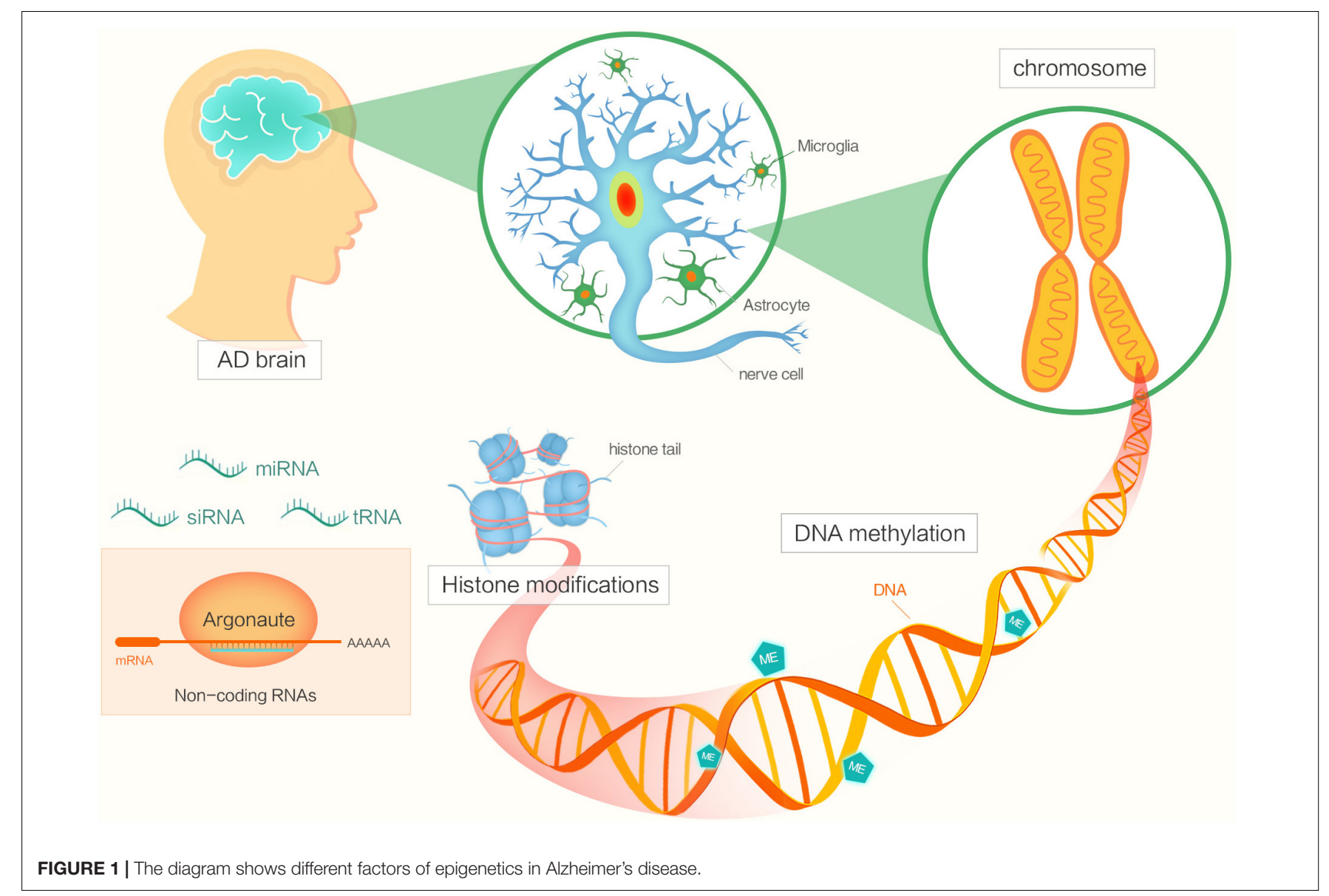

differential methylation dynamics through DNA methylation analysis on purified neurons and glia cells (Gasparoni et al., 2018). Differences were found in DNA methylation in differentiated human neurons with and without $A \beta$ treatment, and that $A \beta$ could change the DNA methylation status of some cell-fate genes (Taher et al., 2014).

\section{CHROMATIN REMODELING AND HISTONE MODIFICATIONS}

Chromatin is an assemblage of genomic DNA, histone proteins and associated factors. Chromatin can be dynamically altered by various modifications of histones. Other possible modifications include nucleosome repositioning, chromatin remodeling, and nuclear compartmentalization (Qureshi and Mehler, 2010). Histones are basic proteins that form the building blocks of nucleosomes. Their tails are susceptible to various modifications, including lysine $(\mathrm{K})$, arginine $(\mathrm{R})$, and histidine $(\mathrm{H})$ methylation (Lardenoije et al., 2015). These alterations together constitute the so-called histone code. The histone code is very complex and includes several types of covalent modifications, such as acetylation, methylation, phosphorylation, and ubiquitination, of at least 20 possible sites within the histone proteins (Jenuwein and Allis, 2001).
Among all the above modifications, acetylation is certainly the best characterized post-translational modification of core histones. Histone acetylation is catalyzed by histone acetyltransferase (HAT) while deacetylation is influenced by histone deacetylase (HDAC). It is known that acetylation of histones is associated with enhanced rates of gene transcription, while deacetylation represses gene expression by condensing the chromatin. Here, we want to discuss the role played by HDAC in the AD mechanisms.

Class I HDACs, such as HDAC2 and HDAC3, are expressed at much higher levels than the other HDACs in the memoryassociated regions of the brain (Datta et al., 2018). A recent study used a mouse model of $\mathrm{AD}$, which deleted the HDAC1 and HDAC2 genes in microglia cells, leading to a decrease in amyloid load and improved cognitive impairment by enhancing microglial amyloid phagocytosis (Broide et al., 2007). In the CK-p25 AD mouse model, Graff et al. (2012) found that elevated HDAC2 levels epigenetically block the expression of neuroplasticity genes during neurodegeneration, and HDAC2 reduces the histone acetylation of genes important for learning and memory. In another AD mouse model, HDAC2 was found to be strongly expressed in the hippocampus and prefrontal cortex. Neuron-specific overexpression of HDAC2 led to a decrease of dendritic spine density, synapse number, synaptic plasticity and memory formation (Levenson et al., 2004; Guan et al., 2009). In 
conditions of stress and injury, the level of HDAC2 increases, and this causes decreased expression of genes related to memory and cognition (Levenson et al., 2004; Graff et al., 2012). HDAC3 also plays a vital role in regulating long-term memory formation. Deletion of HDAC3 in the dorsal hippocampus leads to enhanced long-term memory for object location (Mcquown et al., 2011).

In addition to class I HDACs, there is some evidence that class II HDACs are involved to some degree in AD. Class II HDACs are subdivided into class IIa and class IIb (Haggarty and Tsai, 2011). HDAC6, a class IIb HDAC, is elevated by $52 \%$ and $91 \%$ in the cortex and hippocampus of patients with AD. HDAC6 co-localizes with tau protein in the AD hippocampus, and tau phosphorylation can be decreased by reducing HDAC6 levels (Ding et al., 2008). Govindarajan et al. (2013) using a mouse model of $\mathrm{AD}$, found that decreasing HDAC6 levels could improve cognitive impairment. HDAC4, a member of class IIa, is reported to be associated with synaptic plasticity and memory formation, and loss of it is detrimental to learning and memory (Kim et al., 2012). D'Addario et al. (2017) found that in the peripheral blood cells of monozygotic twins discordant for $\mathrm{AD}$, higher levels of gene expression of HDAC2 and HDAC9 were found in the AD twin. Recently, it was reported that HDAC2 dysregulation could contribute to cholinergic nucleus basalis of Meynert (nbM) neuronal dysfunction, NFT pathology, and cognitive decline during clinical progression of AD (Mahady et al., 2018).

The class III HDACs are called sirtuins, SIRT1-7 (Gray and Ekstrom, 2001). The level of SIRT1 is reduced in the $\mathrm{AD}$ cortex, and SIRT1 levels are negatively correlated with the accumulation of paired helical tau filaments (Julien et al., 2009). Tau acetylation can promote pathological tau aggregation, while SIRT1 causes tau deacetylation. There is extensive evidence that SIRT1 reduction is involved in $\mathrm{AD}$ tau pathology, and tau acetylation of lysine 28 inhibits tau function via impaired tau-microtubule interactions, promoting tau aggregation (Cohen et al., 2011). Moreover, a significant positive correlation between SIRT1 levels and dementia was found whereby dementia risk increases by a factor of 1.16 due to an increase in the SIRT1 level and a factor of 24.23 due to a decrease in the TLR4 level (Kilic et al., 2018).

Histone acetyltransferases are also involved in the regulation of AD neurogenesis. For example, Tip60, a type of histone acetyltransferase, epigenetically regulates genes enriched in neurons, and its function in axonal transport is mediated by APP. More importantly, Tip60 plays a neuroprotective role in APP-induced axonal transport and functional locomotion defects (Johnson et al., 2013).

In conclusion, a range of studies indicates that histone modifications play a vital role in the development of AD. HDACs can both promote and impair memory formation and cognition.

\section{NON-CODING RNA REGULATION}

Non-coding RNAs (ncRNAs) can cause changes in gene expression and the production of proteins and they are involved in the pathology of many neurodegenerative diseases. A recent study used microarray analysis to characterize the expression patterns of circular RNAs (circRNAs), miRNAs and mRNAs in hippocampal tissue from an $A \beta 1-42$-induced $A D$ rat model, and found that a total of 555 circRNAs, 183 miRNAs, and 319 mRNAs were significantly dysregulated (change $\geq 2$-fold and $p$-value < 0.05) in the hippocampus (Wang et al., 2018). In this section, we discuss three aspects of the relationship between ncRNAs and AD.

\section{MiRNAS IN AD}

In $\mathrm{AD}$, miRNAs are expressed differently in the postmortem brain, blood monocytes and cerebrospinal fluid (CSF). The expression of miRNAs in the hippocampus, medial frontal gyrus and CSF is regionally and stage-specifically altered in AD patient brains using a sensitive qRT-PCR platform, and miRNAs are involved in pathways related to amyloid processing, neurogenesis, insulin resistance, and innate immunity in AD pathogenesis (Cogswell et al., 2008). Levels of members of the miR-29 family, including miR-29a, miR-29b, and miR$29 \mathrm{c}$, are decreased in the brains of patients with $\mathrm{AD}$, and this is related to high BACE1 protein expression (Hebert et al., 2008). It has been reported that miRNA-7, miRNA-9-1, miRNA-23a/miRNA-27a, miRNA-34a, miRNA-125b-1, miRNA146a, and miRNA-155 are significantly increased in abundance in $\mathrm{AD}$-affected superior temporal lobe neocortexes (Brodmann area 22). These seven upregulated miRNAs are involved in the coordination of amyloid production and clearance (Pogue and Lukiw, 2018). Higaki et al. (2018) suggested that miR-200b/c reduces $A \beta$ secretion and $A \beta$-induced cognitive impairment by promoting insulin signaling. Moreover, exosome miR-135a and miR-384 were found to be upregulated, while miR-193b was downregulated, in the serum of $\mathrm{AD}$ patients compared to that of normal controls (Yang et al., 2018). A study using Tg2576 AD transgenic mice and human AD brain samples found that miR-206 could regulate brain-derived neurotrophic factor (BDNF), which regulates synaptic plasticity and memory (Lee et al., 2012). Moreover, of 8098 individually measured miRNAs in blood cells, six of these miRNAs such as miR-107, miR-125b, miR-146a, miR-181c, miR-29b, and miR-342 were found to be significantly downregulated in individuals with AD compared to controls (Fransquet and Ryan, 2018). These findings indicate that miRNAs are potential biomarkers for $\mathrm{AD}$, and that miRNAs might be involved in the pathophysiology of $\mathrm{AD}$.

\section{LONG NON-CODING RNAs IN AD}

Long non-coding RNAs (lncRNA) also participate in the pathology of $\mathrm{AD}$. Using a microarray analysis, it was found that in the hippocampal tissue of a rat model of $\mathrm{AD}$, a total of 315 lncRNAs and 311 mRNAs were significantly dysregulated ( $\geq 2$ fold, $p<0.05$ ) (Yang et al., 2017). A type of ncRNA called 51A has been found to regulate the SORL1 gene, which encodes a sorting receptor for the APP holoprotein (Rogaeva et al., 2007). Another 
study concluded that $51 \mathrm{~A}$ might increase AD susceptibility by increasing APP formation (Ciarlo et al., 2013). Other lncRNAs are also related to $\mathrm{AD}$ mechanisms. For example, RNA 17A was found to be increased in $\mathrm{AD}$. This lncRNA regulates GABAB receptor alternative splicing and signaling in response to inflammatory stimuli (Massone et al., 2011). NDM29 RNA upregulation is accompanied by altered APP modulation and can promote the cleavage activities of BACE (Massone et al., 2012). BC200 RNA is reported to be highly increased in the AD brain in parallel with disease progression (Wu et al., 2013). BC1, another type of lncRNA, is found to induce APP mRNA translation via association with fragile $\mathrm{X}$ syndrome protein (FMRP) in mouse model AD brains (Zhang T. et al., 2017). It was recently reported that EBF3 knockdown can reverse A $\beta 25$-35-induced apoptosis in SH-SY5Y cells, and that lncRNA EBF3-AS promotes neuron apoptosis in $\mathrm{AD}$ through regulation of $\mathrm{EBF} 3$ expression ( $\mathrm{Gu}$ et al., 2018). Furthermore, the overexpression of $A \beta$ and $A \beta-42$ in $A D$ could increase BACE1 antisense transcript lncRNA levels, leading to amyloid protein aggregation (Faghihi et al., 2008).

\section{OTHER ncRNAs IN AD}

In addition to miRNA and IncRNA, there are other classes of ncRNAs that play roles in $\mathrm{AD}$ that are not as well understood. Recently, it was reported that circRNA-associatedceRNA networks in an AD mouse model are involved in $\mathrm{A} \beta$ clearance and myelin function (Zhang S. et al., 2017). For example, circRNA CIRS-7 significantly decreases in brains with $\mathrm{AD}$. Its effect is to reduce the protein levels of APP and BACE1 (Lukiw, 2013). Y RNA is reported to play an essential pathological role in the altered mRNA landscape of AD (Champagne et al., 2001). Another interesting ncRNA is small NRSE dsRNA, which is a 20bp double-stranded RNA. NRSE dsRNA has been found to bind to the REST complex and activate it. The REST complex protects neurons from oxidative stress and amyloid $\beta$-protein toxicity (Rougeulle et al., 1998). The U1 snRNP is reported to show very high expression in neurofibrillary tangles in sporadic AD and early-onset AD (Hales et al., 2014a,b). All these findings point to ways that ncRNAs may take part in the development of $\mathrm{AD}$.

\section{APPLICATIONS OF EPIGENETICS TO AD THERAPEUTICS}

\section{DNA Methylation-Based Therapy}

It has been shown that hypomethylation of AD risk genes such as APP, PSEN1, and PSEN2 has effects on learning and memory. Studies have shown that increases in methyl donor S-adenosylL-methionine (SAM) can decrease APP and PSEN1 expression by promoter hypermethylation (Scarpa et al., 2003; Fuso et al., 2005). It may also be relevant that the impaired learning ability induced by lead can be improved significantly by SAM, perhaps by ameliorating the impairments in the long-term potentiation of excitatory postsynaptic potentials that are caused by lead (Cao et al., 2008). Moreover, increasing levels of B12, folate and other methionine sources in the diet can promote methionine bioavailability and reverse elevated expression of APP and PSEN1 (Chan and Shea, 2007; Fuso et al., 2008).

Several studies have focused on finding DNMT inhibitors that could modulate the methylation of AD risk genes. DNMT inhibitors such as azacitidine and decitabine have been approved by the FDA for used in tumors and leukemia (Momparler et al., 1997; Issa et al., 2004). This approach has also been used in some other neurodegenerative diseases, including Friedreich's ataxia and fragile X syndrome (Chiurazzi et al., 1998; Sandi et al., 2013).

It has also been found that DNA methylation is related to the state of NSC differentiation. As a result, the use of the DNA methylation inhibitor 5-aza-cytidine to treat NSCs could alter DNA methylation, thereby disrupting migration and differentiation (Singh et al., 2009). Loss of Dnmt3a, a type of DNA methyltransferase, was found to impair hematopoietic stem cell differentiation (Challen et al., 2011). Another study showed that intrinsic epigenetic mechanisms play critical roles in the regulation of adult NSC functions. Using methylated CpG binding protein 1 (Mbd1)-deficient mice, it was found that both acute knockdown of Mbd1 or overexpression of fibroblast growth factor 2 (Fgf-2) in adult neural stem and progenitor cells inhibited neuronal differentiation (Li et al., 2008).

\section{Histone Modification-Based Therapy}

Histone deacetylase inhibitors, including valproic acid (VPA), sodium 4-phenylbutyrate (4-PBA), vorinostat (SAHA), trichostatin A (TSA) and nicotinamide, have been shown to produce good results in $\mathrm{AD}$ mouse models. We discuss these five inhibitors separately.

Valproic acid has been shown to inhibit $A \beta$ production in a transgenic AD mouse model (Su et al., 2004). In neuroblastoma and glioma cell lines, VPA could prevent amyloid- $\beta$ aggregation by increasing clusterin expression (Nuutinen et al., 2010). VPA was also found to decrease $A \beta$ production in transgenic mice, improving their behavioral deficits (Qing et al., 2008). In an AD mouse model it relieved contextual memory deficits via histone H4 acetylation (Kilgore et al., 2010).

4 -PBA is reported to decrease $\beta$-amyloid production and reverse spatial learning and memory deficits. In the Tg2576 AD mouse model, administration of 4-PBA led to a decrease in tau phosphorylation by increasing GSK-3 $\beta$ (Ricobaraza et al., 2009) as well as increasing intraneuronal $A \beta$ clearance and restoration of dendritic spine densities in hippocampal CA1 pyramidal neurons (Ricobaraza et al., 2012).

SAHA is found to increase the H4K12 acetylation level, thus restoring learning ability in a mouse model (Peleg et al., 2010). In an astrocyte cell line, it induced the expression of clusterin, which might prevent the progress of AD pathogenesis (Nuutinen et al., 2010). In the APPswe/PS1dE9 AD mouse model, SAHA was reported to restore contextual memory by inhibiting HDAC6 in the early stage of AD (Kilgore et al., 2010).

Trichostatin A is found to induce brain-derived neurotrophic factor (BDNF) exon I-IX mRNA in Neuro-2a cells (Ishimaru et al., 2010). Another study of hippocampal neurons also suggested that TSA treatment could be useful at promoter 1 by acetylating histones (H3AcK9/K14) (Tian et al., 2010). 
Nicotinamide was found to restore cognitive deficits. Nicotinamide can also increase the acetylation of alpha-tubulin and MAP2c, thereby increasing microtubule stability. These observations suggest a possibility that nicotinamide could be useful in treating AD (Green et al., 2008).

\section{ncRNA Regulation-Based Therapy}

Non-coding RNAs are widely expressed in the brain and show a range of differences between $\mathrm{AD}$ and healthy controls. Numerous studies have focused on finding potential treatments using ncRNAs. Studies have found that overexpression of miR-124 and miR-195 could down-regulate A $\beta$ levels by controlling BACE1 gene expression (Fang et al., 2012; Zhu et al., 2012). It is reported that DNA antisense oligonucleotides can hasten the degradation of UBE3A-ATS, which might be an avenue of treatment for AD (Meng et al., 2015). In Tg-19959 mice, knockdown of BACE1 or BACE1-AS transcripts causes reductions in BACE1 protein and insoluble $A \beta$ (Modarresi et al., 2011). In an in vitro preparation, MiR-16 was found to be an effective inhibitor of amyloid precursor protein (APP) and BACE1 expression, A $\beta$ peptide production, and tau phosphorylation (Parsi et al., 2015).

miRNA regulators have also been evaluated for use in treating AD. For example, a type of neutralizing inhibitor of miR-206 called AM206 was injected into the cerebral ventricles of an $\mathrm{AD}$ mouse model and found to improve memory function (Lee et al., 2012) as well as improving hippocampal neurogenesis and synaptic density. It was reported that RNA interference-mediated knockdown of the long-form phosphodiesterase-4D (PDE4D) enzyme could reverse memory deficits caused by accumulation of amyloid-beta (42) (Zhang et al., 2014). Another study found that miR-34c levels were increased in the hippocampus of $\mathrm{AD}$ patients, leading to a suggestion that it might be a biomarker for $\mathrm{AD}$. In an $\mathrm{AD}$ mouse model, targeting miR-34 was found to lead to the recovery of learning ability (Zovoilis et al., 2011).

\section{DISCUSSION AND FUTURE PERSPECTIVES}

Alzheimer's disease is a progressive neurodegenerative disease which is already a substantial burden on the world. The mechanisms of $\mathrm{AD}$ are complex and have been the subject of many theories. We have gained insight into the role of epigenetic modulation, which comprises DNA methylation, histone modification and the effects of ncRNAs. While many differences in these three kinds of epigenetic regulation have been observed between AD patients or models and controls, much work will still be needed to understand this topic and to find potential targets for the treatment of $\mathrm{AD}$.

One of the main challenges for epigenetic therapy is to find molecules that can pass through the blood-brain barrier (BBB). In general, only molecules less than 500 daltons, with fewer than 8 pairs of hydrogen bonds, can move through the $\mathrm{BBB}$. There is a variety of ways of working around this limitation, including $\mathrm{BBB}$ disruption, intracerebral implantation and intracerebroventricular infusion (Pardridge, 2005). As discussed above, DNMT inhibitors and HDAC inhibitors are potential therapeutic drugs, and these two kinds of molecules are able to cross the BBB (Hockly et al., 2003; Rai et al., 2008; Kwa et al., 2011). However, several additional factors need to be considered, including efficacy, toxicity, delivery and patient factors. Even though DNMT inhibitors are widely used in treating tumors and leukemia, they are not yet suitable for continuous treatment of AD. Because of their genotoxicity and low stability, they remain at the preclinical stage of development (Cuadrado-Tejedor et al., 2013; Erdmann et al., 2015). However, the clinical side effects of HDAC inhibitors are well known (Subramanian et al., 2010). As an example, the HDAC inhibitor SAHA can induce reactive oxygen species and DNA damage in acute myeloid leukemia cells (Petruccelli et al., 2011). Therefore, more detailed studies are needed to develop safe and effective treatments using DNMT inhibitors and HDAC inhibitors.

Other approaches to ncRNA-based therapy has been examined in recent years. A study showed that a short peptide derived from rabies virus glycoprotein (RVG) assists the transvascular delivery of small interfering RNA (siRNA) to the brain, leading to specific gene silencing inside the brain. Intravenous treatment with RVG9R-bound antiviral siRNA afforded robust protection against fatal viral encephalitis in mice (Kumar et al., 2007). Other studies have shown that nanoparticle carriers may be a useful method of moving drugs through the BBB (Kreuter, 2001). All of these are methods that may be useful for implementing small interfering RNA therapy.

\section{CONCLUSION}

In conclusion, we have discussed various epigenetic approaches to the treatment of $\mathrm{AD}$, and new methods have emerged to aid in validating epigenetic targets. However, careful characterization of chemical probes is essential to ensure accurate biological conclusions. A new generation of selective chemical probes needs to be developed to reduce side effects. Studies on epigenetics may lead researchers to new methods of AD diagnosis and therapy. Even though the transition from bench to bedside may be challenging in the near future, current research strongly suggests that epigenetic regulation will become an important tool in $\mathrm{AD}$ treatment.

\section{AUTHOR CONTRIBUTIONS}

XL contributed to the central idea and wrote the initial draft of the manuscript. BJ and LS contributed to refining the ideas, carrying out additional modifications, and finalizing the manuscript. All authors contributed to writing and revising the manuscript.

\section{FUNDING}

This study was supported by the National Natural Science Foundation of China (No. 81701134 to BJ) and Youth Research Foundation of Xiangya Hospital, Central South University (No. 2016Q01 to BJ). 


\section{REFERENCES}

Blanco-Luquin, I., Altuna, M., Sanchez-Ruiz, D. G. J., Urdánoz-Casado, A., Roldán, M., Cámara, M. et al. (2018). PLD3 epigenetic changes in the hippocampus of Alzheimer's disease. Clin Epigenet. 10:116. doi: 10.1186/s13148-0180547-3

Broide, R. S., Redwine, J. M., Aftahi, N., Young, W., Bloom, FE., and Winrow, CJ. (2007). Distribution of histone deacetylases 1-11 in the rat brain. J. Mol. Neurosci. 31, 47-58. doi: 10.1007/BF02686117

Cao, X J., Huang, S. H., Wang, M., Chen J. T, and Ruan, D. Y. (2008). S-adenosyl-Lmethionine improves impaired hippocampal long-term potentiation and water maze performance induced by developmental lead exposure in rats. Eur. J. Pharmacol. 595, 30-34. doi: 10.1016/j.ejphar.2008.07.061

Challen, G. A., Sun, D., Jeong, M., Luo, M, Jelinek, J, Berg J. S.et al. (2011). Dnmt3a is essential for hematopoietic stem cell differentiation. Nat. Genet. 44, 23-31. doi: 10.1038/ng.1009

Champagne, F., Diorio, J., Sharma, S., and Meaney M. J. (2001). Naturally occurring variations in maternal behavior in the rat are associated with differences in estrogen-inducible central oxytocin receptors. Proc. Natl. Acad. Sci. U.S.A. 98, 12736-12741. doi: 10.1073/pnas.221224598

Champagne, F. A. (2013). Epigenetics and developmental plasticity across species. Dev. Psychobiol. 55, 33-41. doi: 10.1002/dev.21036

Chan, A, and Shea, T. B. (2007). Folate deprivation increases presenilin expression, gamma-secretase activity, and Abeta levels in murine brain: potentiation by ApoE deficiency and alleviation by dietary S-adenosyl methionine. J. Neurochem. 102, 753-760. doi: 10.1111/j.1471-4159.2007.04589.x

Chiurazzi, P., Pomponi, M. G., Willemsen, R., Oostra BA, and Neri G. (1998). In vitro reactivation of the FMR1 gene involved in fragile $\mathrm{X}$ syndrome. Hum. Mol. Genet. 7, 109-113. doi: 10.1093/hmg/7.1.109

Chouliaras, L., Mastroeni, D., Delvaux, E., Grover, A., Kenis, G., Hof, P. R., et al. (2013). Consistent decrease in global DNA methylation and hydroxymethylation in the hippocampus of Alzheimer's disease patients. Neurobiol. Aging 34, 2091-2099. doi: 10.1016/j.neurobiolaging.2013.02.021

Ciarlo, E., Massone, S., Penna, I., Nizzari, M., Gigoni, A., Dieci, G., et al. (2013). An intronic ncRNA-dependent regulation of SORL1 expression affecting Abeta formation is upregulated in post-mortem Alzheimer's disease brain samples. Dis. Model. Mech. 6, 424-433. doi: 10.1242/dmm.009761

Cogswell, J. P., Ward, J., Taylor, I. A., Waters, M., Shi, Y., Cannon, B., et al. (2008). Identification of miRNA changes in Alzheimer's disease brain and CSF yields putative biomarkers and insights into disease pathways. J. Alzheimers Dis. 14, 27-41. doi: 10.3233/JAD-2008-14103

Cohen, T. J., Guo, J. L., Hurtado, D. E., Kwong, L. K., Mills, I. P., Trojanowski, J. Q., et al. (2011). The acetylation of tau inhibits its function and promotes pathological tau aggregation. Nat. Commun. 2:252. doi: 10.1038/ncomms 1255

Condliffe, D., Wong, A., Troakes, C., Proitsi, P., Patel, Y., Chouliaras, L., et al. (2014). Cross-region reduction in 5-hydroxymethylcytosine in Alzheimer's disease brain. Neurobiol. Aging 35, 1850-1854. doi: 10.1016/j.neurobiolaging. 2014.02.002

Cuadrado-Tejedor, M., Oyarzabal, J., and Lucas, M. P, and García-Osta. (2013). Epigenetic drugs in Alzheimer's disease. Biomol. Concepts 4, 433-445. doi: 10. 1515/bmc-2013-0012

D’Addario, C., Candia, S. B., Arosio, B., Di Bartolomeo, M., Abbate, C., Casè A., et al. (2017). Transcriptional and epigenetic phenomena in peripheral blood cells of monozygotic twins discordant for alzheimer's disease, a case report. J. Neurol. Sci. 372, 211-216. doi: 10.1016/j.jns.2016.11.052

Datta, M., Staszewski, O., Raschi, E., Frosch, M., Hagemeyer, N., Tay, T. L., et al. (2018). Histone deacetylases 1 and 2 regulate microglia function during development, homeostasis, and neurodegeneration in a contextdependent manner. Immunity 48, 514-529. doi: 10.1016/j.immuni.2018. 02.016

Di Francesco, A., Arosio, B., Falconi, A., Micioni Di Bonaventura, M. V., Karimi, M., Mari, D., et al. (2015). Global changes in DNA methylation in Alzheimer's disease peripheral blood mononuclear cells. Brain Behav. Immun. 45, 139-144. doi: 10.1016/j.bbi.2014.11.002

Ding, H., Dolan, P. J., and Johnson, G. V. (2008). Histone deacetylase 6 interacts with the microtubule-associated protein tau. J. Neurochem. 106, 2119-2130. doi: $10.1111 /$ j.1471-4159.2008.05564.x
Diniz, L. P., Tortelli, V., Matias, I., Morgado, J., Bérgamo Araujo, A. P., Melo, H. M., et al. (2017). Astrocyte transforming growth factor beta 1 protects synapses against Abeta oligomers in Alzheimer's disease model. J. Neurosci. 37, 6797-6809. doi: 10.1523/JNEUROSCI.3351-16.2017

Erdmann, A., Halby, L., Fahy, J., and Arimondo, P. B. (2015). Targeting DNA methylation with small molecules: what's next? J. Med. Chem. 58, 2569-2583. doi: 10.1021/jm500843d

Faghihi, M. A., Modarresi, F., Khalil, A. M., Wood, D. E, Sahagan, B. G., Morgan, T. E., et al. (2008). Expression of a noncoding RNA is elevated in Alzheimer's disease and drives rapid feed-forward regulation of beta-secretase. Nat. Med. 14, 723-730. doi: 10.1038/nm1784

Fang, M., Wang, J., Zhang, X., Geng, Y., Hu, Z., Rudd, J. A., et al. (2012). The miR124 regulates the expression of BACE1/beta-secretase correlated with cell death in Alzheimer's disease. Toxicol. Lett. 209, 94-105. doi: 10.1016/j.toxlet.2011. 11.032

Fransquet, P. D, and Ryan, J. (2018). Micro RNA as a potential bloodbased epigenetic biomarker for Alzheimer's disease. Clin. Biochem. 58, 5-14. doi: 10.1016/j.clinbiochem.2018.05.020

Fuso, A., Nicolia, V., Cavallaro, R. A., Ricceri, L., D’Anselmi, F., Coluccia, P., et al. (2008). B-vitamin deprivation induces hyperhomocysteinemia and brain S-adenosylhomocysteine, depletes brain S-adenosylmethionine, and enhances PS1 and BACE expression and amyloid-beta deposition in mice. Mol. Cell Neurosci. 37, 731-746. doi: 10.1016/j.mcn.2007.12.018

Fuso, A., Seminara, L., Cavallaro, R. A., D'Anselmi, F, and Scarpa, S. (2005). S-adenosylmethionine/homocysteine cycle alterations modify DNA methylation status with consequent deregulation of PS1 and BACE and betaamyloid production. Mol. Cell Neurosci. 28, 195-204. doi: 10.1016/j.mcn.2004. 09.007

Gasparoni, G., Bultmann, S., Lutsik, P., Kraus, T. F. J., Sordon, S., Vlcek, J., et al. (2018). DNA methylation analysis on purified neurons and glia dissects age and Alzheimer's disease-specific changes in the human cortex. Epigenet. Chromatin. 11:41. doi: 10.1186/s13072-018-0211-3

Govindarajan, N., Rao, P., Burkhardt, S, Sananbenesi, F., Schlüter, O. M., Bradke, F., et al. (2013). Reducing HDAC6 ameliorates cognitive deficits in a mouse model for Alzheimer's disease. EMBO Mol. Med. 5, 52-63. doi: 10.1002/ emmm.201201923

Graff, J., Rei, D., Guan, J. S., Wang, W. Y., Seo, J., Hennig, K. M., et al. (2012). An epigenetic blockade of cognitive functions in the neurodegenerating brain. Nature 483, 222-226. doi: 10.1038/nature10849

Gray, S. G, and Ekstrom, T. J. (2001). The human histone deacetylase family. Exp. Cell Res. 262, 75-83. doi: 10.1006/excr.2000.5080

Green, K. N., Steffan, J. S., Martinez-Coria, H., Sun, X., Schreiber, S. S., and Thompson, L. M. (2008). Nicotinamide restores cognition in Alzheimer's disease transgenic mice via a mechanism involving sirtuin inhibition and selective reduction of Thr231-phosphotau. J. Neurosci. 28, 11500-11510. doi: 10.1523/JNEUROSCI.3203-08.2008

Gu, C., Chen, C., Wu, R., Dong, T., Hu, X., Yao, Y., et al. (2018). Long noncoding RNA EBF3-as promotes neuron apoptosis in Alzheimer's Disease. DNA Cell Biol. 37, 220-226. doi: 10.1089/dna.2017.4012

Guan, J. S., Haggarty, S. J., Giacometti, E., Dannenberg. J. H., Joseph, N., Gao, J., et al. (2009). HDAC2 negatively regulates memory formation and synaptic plasticity. Nature 459, 55-60. doi: 10.1038/nature07925

Haggarty, S. J., and Tsai, L. H. (2011). Probing the role of HDACs and mechanisms of chromatin-mediated neuroplasticity. Neurobiol. Learn. Mem. 96, 41-52. doi: 10.1016/j.nlm.2011.04.009

Hales, C. M., Dammer, E. B., Diner, I., Yi, H, Seyfried, N. T, Gearing, M., et al. (2014a). Aggregates of small nuclear ribonucleic acids (snRNAs) in Alzheimer's disease. Brain Pathol. 24, 344-351. doi: 10.1111/bpa. 12133

Hales, C. M., Seyfried, N. T., Dammer, E. B., Duong, D., Yi, H., Gearing, M., et al. (2014b). U1 small nuclear ribonucleoproteins (snRNPs) aggregate in Alzheimer's disease due to autosomal dominant genetic mutations and trisomy 21. Mol. Neurodegener. 9:15. doi: 10.1186/1750-1326-9-15

Hebert, S. S., Horre, K., Nicolai, L., Papadopoulou, A. S., Mandemakers, W., Silahtaroglu, A. N., et al. (2008). Loss of microRNA cluster miR-29a/b-1 in sporadic Alzheimer's disease correlates with increased BACE1/beta-secretase expression. Proc. Natl. Acad. Sci. U.S.A. 105, 6415-6420. doi: 10.1073/pnas. 0710263105 
Higaki, S., Muramatsu, M., Matsuda, A., Matsumoto, K, Satoh, J. I., Michikawa, M., et al. (2018). Defensive effect of microRNA-200b/c against amyloid-beta peptide-induced toxicity in Alzheimer's disease models. PLoS One. 13:e196929. doi: 10.1371/journal.pone.0196929

Hockly, E., Richon, V. M., Woodman, B., Smith, D. L., Zhou, X., Rosa, E., et al. (2003). Suberoylanilide hydroxamic acid, a histone deacetylase inhibitor, ameliorates motor deficits in a mouse model of Huntington's disease. Proc. Natl. Acad. Sci. U.S.A. 100, 2041-2046. doi: 10.1073/pnas.043787 0100

Holliday, R. (2006). Epigenetics: a historical overview. Epigenetics 1, 76-80. doi: 10.4161/epi.1.2.2762

Ishimaru, N., Fukuchi, M., Hirai, A., Chiba, Y., Tamura, T., Takahashi, N. et al. (2010). Differential epigenetic regulation of BDNF and NT-3 genes by trichostatin A and 5-aza-2'-deoxycytidine in Neuro-2a cells. Biochem. Biophys. Res. Commun. 394, 173-177. doi: 10.1016/j.bbrc.2010.02.139

Issa, J. P., Garcia-Manero, G., Giles, F. J., Mannari, R., Thomas, D., Faderl, S., et al. (2004). Phase 1 study of low-dose prolonged exposure schedules of the hypomethylating agent 5-aza-2'-deoxycytidine (decitabine) in hematopoietic malignancies. Blood 103, 1635-1640. doi: 10.1182/blood-2003-030687

Jenuwein, T., and Allis, C. D. (2001). Translating the histone code. Science 293, 1074-1080. doi: 10.1126/science.1063127

Johnson, A. A, Sarthi, J., Pirooznia, S. K, Reube, W., and Elefant, F. (2013). Increasing Tip60 HAT levels rescues axonal transport defects and associated behavioral phenotypes in a Drosophila Alzheimer's disease model. J. Neurosci. 33, 7535-7547. doi: 10.1523/JNEUROSCI.3739-12.2013

Jones, P. A., and Laird, P. W. (1999). Cancer epigenetics comes of age. Nat. Genet. 21, 163-167. doi: 10.1038/5947

Julien, C., Tremblay, C., Emond, V., Lebbadi, M., Salem, N. Jr., Bennett, D. A., et al. (2009). Sirtuin 1 reduction parallels the accumulation of tau in Alzheimer disease. J. Neuropathol. Exp. Neurol. 68, 48-58. doi: 10.1097/NEN. ob013e3181922348

Kemme, C. A., Marquez, R., Luu, R. H., and Iwahara, J. (2017). Potential role of DNA methylation as a facilitator of target search processes for transcription factors through interplay with methyl-CpG-binding proteins. Nucleic Acids Res. 45:7751-7759. doi: 10.1093/nar/gkx387

Kilgore, M., Miller, C. A., Fass, D. M., Hennig, K. M, Haggarty, S. J, Sweatt, J. D. et al. (2010). Inhibitors of class 1 histone deacetylases reverse contextual memory deficits in a mouse model of Alzheimer's disease. Neuropsychopharmacology 35, 870-880. doi: 10.1038/npp.2009.197

Kilic, U., Elibol, B., Uysal, O., Kilic E., Yulug, B., Sayin Sakul A., et al. (2018). Specific alterations in the circulating levels of the SIRT1, TLR4, and IL7 proteins in patients with dementia. Exp. Gerontol. 111, 203-209. doi: 10.1016/j.exger. 2018.07.018

Kim, M. S., Akhtar, M. W., Adachi, M., Mahgoub M, Bassel-Duby R, Kavalali ET., et al. (2012). An essential role for histone deacetylase 4 in synaptic plasticity and memory formation. J. Neurosci. 32, 10879-10886. doi: 10.1523/JNEUROSCI. 2089-12.2012

Kobayashi, N., Shinagawa, S., Nagata, T., Shimada, K., Shibata, N., Ohnuma, T., et al. (2016). Usefulness of DNA methylation levels in COASY and SPINT1 gene promoter regions as biomarkers in diagnosis of Alzheimer's disease and amnestic mild cognitive impairment. PLoS One 11:e168816. doi: 10.1371/ journal.pone.0168816

Kreuter, J. (2001). Nanoparticulate systems for brain delivery of drugs. Adv. Drug Deliv. Rev. 47, 65-81. doi: 10.1016/S0169-409X(00)00 122-8

Kumar, P., Wu, H., Mcbride, J. L., Jung, K. E., Kim, M. H., Davidson, B. L., et al. (2007). Transvascular delivery of small interfering RNA to the central nervous system. Nature 448, 39-43. doi: 10.1038/nature05901

Kwa, F. A., Balcerczyk, A., Licciardi, P., El-Osta, A., and Karagiannis, T. C. (2011). Chromatin modifying agents - the cutting edge of anticancer therapy. Drug Discov. Today 16, 543-547. doi: 10.1016/j.drudis.2011. 05.012

Lane-Donovan, C., and Herz, J. (2017). ApoE, ApoE receptors, and the synapse in Alzheimer's disease. Trends Endocrinol. Metab. 28, 273-284. doi: 10.1016/j.tem. 2016.12.001

Lansdall, C. J., Coyle-Gilchrist, I., Jones, P. S., Vázquez Rodríguez, P., Wilcox, A., Wehmann, E., et al. (2017). Apathy and impulsivity in frontotemporal lobar degeneration syndromes. Brain 140, 1792-1807. doi: 10.1093/brain/ awx101

Lardenoije, R., Iatrou, A., Kenis, G., Kompotis, K., Steinbusch, H. W., Mastroeni, D., et al. (2015). The epigenetics of aging and neurodegeneration. Prog. Neurobiol. 131, 21-64. doi: 10.1016/j.pneurobio.2015.05.002

Lee, S. T., Chu, K., Jung, K. H., Kim, J. H., Huh, J. Y., Yoon, H., et al. (2012). miR206 regulates brain-derived neurotrophic factor in Alzheimer disease model. Ann. Neurol. 72, 269-277. doi: 10.1002/ana.23588

Levenson, J. M., O’Riordan, K. J., Brown, K. D., Trinh, M. A., Molfese, D. L., and Sweatt, J. D. (2004). Regulation of histone acetylation during memory formation in the hippocampus. J. Biol. Chem. 279, 40545-40559. doi: 10.1074/ jbc.M402229200

Levy, E., Carman, M. D., Fernandez-Madrid, I. J., Power, M. D., Lieberburg, I., van Duinen, S. G., et al. (1990). Mutation of the Alzheimer's disease amyloid gene in hereditary cerebral hemorrhage, Dutch type. Science 248, 1124-1126. doi: 10.1126/science. 2111584

Li, X., Barkho, B. Z, Luo, Y., Smrt, R. D., Santistevan, N. J., Liu, C., et al. (2008). Epigenetic regulation of the stem cell mitogen Fgf- 2 by Mbd1 in adult neural stem/progenitor cells. J. Biol. Chem. 283, 27644-27652. doi: 10.1074/jbc. M804899200

Lukiw, W. J. (2013). Circular RNA (circRNA) in Alzheimer's disease (AD). Front. Genet. 4:307. doi: 10.3389/fgene.2013.00307.

Mahady, L., Nadeem, M., Malek-Ahmadi, M., Chen, K., Perez, S. E., and Mufson, E. J. (2018). HDAC2 dysregulation in the nucleus basalis of Meynert during the progression of Alzheimer's disease. Neuropathol. Appl. Neurobiol. doi: 10.1111/ nan.12518. [Epub ahead of print].

Mano, T., Nagata, K., Nonaka, T., Tarutani, A., Imamura, T., Hashimoto, T., et al. (2017). Neuron-specific methylome analysis reveals epigenetic regulation and tau-related dysfunction of BRCA1 in Alzheimer's disease. Proc. Natl. Acad. Sci. U.S.A. 114, E9645-E9654. doi: 10.1073/pnas.1707151114

Massone, S., Ciarlo, E., Vella, S., Nizzari, M., Florio, T., Russo, C., et al. (2012). NDM29, a RNA polymerase III-dependent non coding RNA, promotes amyloidogenic processing of APP and amyloid beta secretion. Biochim. Biophys. Acta 1823, 1170-1177. doi: 10.1016/j.bbamcr.2012.05.001

Massone, S., Vassallo, I., Fiorino, G., Castelnuovo, M., Barbieri, F., Borghi, R., et al. (2011). 17A, a novel non-coding RNA, regulates GABA B alternative splicing and signaling in response to inflammatory stimuli and in Alzheimer disease. Neurobiol. Dis. 41, 308-317. doi: 10.1016/j.nbd.2010.09.019

Masters, C. L., Bateman, R., Blennow, K., Rowe, C. C., Sperling, R. A., and Cummings, J. L. (2015). Alzheimer's disease. Nat. Rev. Dis. Primers 1:15056. doi: $10.1038 /$ nrdp. 2015.56

Mastroeni, D., Grover, A., Delvaux, E., Whiteside, C., Coleman, P. D., and Rogers, J. (2010). Epigenetic changes in Alzheimer's disease: decrements in DNA methylation. Neurobiol. Aging 31, 2025-2037. doi: 10.1016/j.neurobiolaging. 2008.12.005

Mastroeni, D., Mckee, A., Grover, A., Rogers, J., and Coleman, P. D. (2009). Epigenetic differences in cortical neurons from a pair of monozygotic twins discordant for Alzheimer's disease. PLoS One 4:e6617. doi: 10.1371/journal. pone.0006617

Mckhann, G. M., Knopman, D. S., Chertkow, H., Hyman, B. T., Jack, C. R Jr., Kawas, C. H., et al. (2011). The diagnosis of dementia due to Alzheimer's disease: recommendations from the National Institute on Aging-Alzheimer's Association workgroups on diagnostic guidelines for Alzheimer's disease. Alzheimers Dement. 7, 263-269. doi: 10.1016/j.jalz.2011.03.005

Mcquown, S. C., Barrett, R. M., Matheos, D. P., Post, R. J., Rogge, G. A., Alenghat, T., et al. (2011). HDAC3 is a critical negative regulator of long-term memory formation. J, Neurosci. 31, 764-774. doi: 10.1523/JNEUROSCI.505210.2011

Mehler, M. F. (2008). Epigenetic principles and mechanisms underlying nervous system functions in health and disease. Prog. Neurobiol. 86, 305-341. doi: 10. 1016/j.pneurobio.2008.10.001

Meng, L., Ward, A. J and Chun, S., Bennett, C. F., Beaudet, A. L., and Rigo, F. (2015). Towards a therapy for Angelman syndrome by targeting a long noncoding RNA. Nature 518, 409-412. doi: 10.1038/nature13975

Mercorio, R., Pergoli, L., Galimberti, D., Favero, C., Carugno, M., Dalla Valle, E., et al. (2018). PICALM Gene Methylation in Blood of Alzheimer's Disease Patients Is Associated with Cognitive Decline. J. Alzheimers Dis. 65, 283-292. doi: $10.3233 /$ JAD- 180242 
Modarresi, F., Faghihi, M. A, Patel, N. S, Sahagan, B. G., and Wahlestedt, C. R. (2011). Knockdown of BACE1-AS nonprotein-coding transcript modulates beta-amyloid-related hippocampal neurogenesis. Int. J. Alzheimers Dis. 2011:929042. doi: 10.4061/2011/929042

Momparler, R. L., Bouffard, D. Y., Momparler, L. F, Dionne J, Belanger K, and Ayoub J. (1997). Pilot phase I-II study on 5-aza-2'-deoxycytidine (Decitabine) in patients with metastatic lung cancer. Anticancer Drugs 8, 358-368. doi: 10.1097/00001813-199704000-00008

Morange, M. (2002). The relations between genetics and epigenetics: a historical point of view. Ann. N.Y.Acad. Sci. 981, 50-60. doi: 10.1111/j.1749-6632.2002. tb04911.x

Nuutinen, T., Suuronen, T., Kauppinen, A., and Salminen, A. (2010). Valproic acid stimulates clusterin expression in human astrocytes: implications for Alzheimer's disease. Neurosci. Lett. 475, 64-68. doi: 10.1016/j.neulet.2010. 03.041

Ozaki, Y., Yoshino, Y., Yamazaki, K., Sao, T., Mori, Y., Ochi, S., et al. (2017). DNA methylation changes at TREM2 intron 1 and TREM2 mRNA expression in patients with Alzheimer's disease. J. Psychiatr. Res. 92, 74-80. doi: 10.1016/j. jpsychires.2017.04.003

Pardridge, W. M. (2005). The blood-brain barrier: bottleneck in brain drug development. NeuroRx 2, 3-14. doi: 10.1602/neurorx.2.1.3

Parsi, S., Smith, P. Y., Goupil, C., Dorval, V., and Hébert, S. S. (2015). Preclinical evaluation of miR-15/107 family members as multifactorial drug targets for Alzheimer's disease. Mol. Ther. Nucleic Acids 4:e256. doi: 10.1038/mtna.2015.33

Peleg, S., Sananbenesi, F., Zovoilis, A., Burkhardt, S., Bahari-Javan, S., Agis-Balboa, R. C. et al. (2010). Altered histone acetylation is associated with age-dependent memory impairment in mice. Science 328, 753-756. doi: 10.1126/science. 1186088

Petruccelli, L. A., Dupere-Richer, D., Pettersson, F., Retrouvey, H., and Skoulikas, S., Miller, W. H. Jr. (2011). Vorinostat induces reactive oxygen species and DNA damage in acute myeloid leukemia cells. PLoS One 6:e20987. doi: 10.1371/journal.pone.0020987

Pogue, A. I, and Lukiw, W. J. (2018). Up-regulated Pro-inflammatory MicroRNAs (miRNAs) in Alzheimer's disease (AD) and Age-Related Macular Degeneration (AMD). Cell Mol. Neurobiol. 38, 1021-1031. doi: 10.1007/s10571-0170572-3

Qing, H., He, G., Ly, P. T., Fox, C. J, Staufenbiel, M., Cai, F., et al. (2008). Valproic acid inhibits Abeta production, neuritic plaque formation, and behavioral deficits in Alzheimer's disease mouse models. J. Exp. Med. 205, 2781-2789. doi: 10.1084 /jem.20081588

Qureshi I A, and Mehler, M. F. (2010). Impact of nuclear organization and dynamics on epigenetic regulation in the central nervous system: implications for neurological disease states. Ann. N.Y. Acad. Sci. 1204(Suppl ), E20-E37. doi: $10.1111 / \mathrm{j} .1749-6632.2010 .05718 . \mathrm{x}$

Rai, M., Soragni, E., Jenssen, K., Burnett, R., Herman, D., Coppola, G., et al. (2008). HDAC inhibitors correct frataxin deficiency in a Friedreich ataxia mouse model. PLoS One 3:e1958. doi: 10.1371/journal.pone. 0001958

Ricobaraza, A., Cuadrado-Tejedor, M., Marco, S., Pérez-Otaño, I., and GarcíaOsta, A. (2012). Phenylbutyrate rescues dendritic spine loss associated with memory deficits in a mouse model of Alzheimer disease. Hippocampus 22, 1040-1050. doi: 10.1002/hipo.20883

Ricobaraza, A., Cuadrado-Tejedor, M., Perez-Mediavilla, A., Frechilla, D., Del Río, J., and García-Osta, A. (2009). Phenylbutyrate ameliorates cognitive deficit and reduces tau pathology in an Alzheimer's disease mouse mode. Neuropsychopharmacology 34, 1721-1732. doi: 10.1038/npp.20 08.229

Rocca, W. A., Petersen, R. C., Knopman, D. S., Hebert, L. E., Evans, D. A., Hall, K. S., et al. (2011). Trends in the incidence and prevalence of Alzheimer's disease, dementia, and cognitive impairment in the United States. Alzheimers Dement. 7, 80-93. doi: 10.1016/j.jalz.2010.11.002

Rogaeva, E., Meng, Y., Lee, J. H., Gu, Y., Kawarai, T., Zou, F., et al. (2007). The neuronal sortilin-related receptor SORL1 is genetically associated with Alzheimer disease. Nat. Genet. 39, 168-177. doi: 10.1038/ng 1943

Rougeulle, C., Cardoso, C., Fontes, M., Colleaux, L., and Lalande M. (1998). An imprinted antisense RNA overlaps UBE3A and a second maternally expressed transcript. Nat. Genet. 19, 15-16. doi: 10.1038/ng0598-15
Sandi, C., Al-Mahdawi, S, and Pook, M. A. (2013). Epigenetics in Friedreich's ataxia: challenges and opportunities for therapy. Genet. Res. Int. 2013:852080. doi: 10.1155/2013/852080

Santana, V. P., Miranda-Furtado, C. L., de Oliveira-Gennaro, F. G., and Dos Reis, R. M. (2017). Genetics and epigenetics of varicocele pathophysiology: an overview. J. Assist. Reprod. Genet. 34, 839-847. doi: 10.1007/s10815-017-0931-5

Scarpa, S., Fuso, A., D’Anselmi, F., and Cavallaro, R. A. (2003). Presenilin 1 gene silencing by S-adenosylmethionine: a treatment for Alzheimer disease?. FEBS Lett. 541, 145-148. doi: 10.1016/S0014-5793(03)00277-1

Selkoe, D. J. (2012). Preventing Alzheimer's disease. Science 337, 1488-1492. doi: $10.1126 /$ science. 1228541

Sery, O., Povova, J., Misek, I., Pešák, L., and Janout, V. (2013). Molecular mechanisms of neuropathological changes in Alzheimer's disease: a review. Folia Neuropathol. 51, 1-9. doi: 10.5114/fn.2013.34190

Shinagawa, S., Kobayashi, N., Nagata, T., Kusaka, A., Yamada, H., Kondo K., et al. (2016). DNA methylation in the NCAPH2/LMF2 promoter region is associated with hippocampal atrophy in Alzheimer's disease and amnesic mild cognitive impairment patients. Neurosci. Lett. 2016, 629, 33-37. doi: 10.1016/j.neulet. 2016.06.055

Singh, R. P., Shiue, K., Schomberg, D., and Zhou, F. C. (2009). Cellular epigenetic modifications of neural stem cell differentiation. Cell Transplant. 18, 1197-1211. doi: 10.3727/096368909X12483162197204

Smith, R. G., Hannon, E., De Jager, P. L., Chibnik, L., Lott, S. J., Condliffe, D., et al. (2018). Elevated DNA methylation across a 48-kb region spanning the HOXA gene cluster is associated with Alzheimer's disease neuropathology. Alzheimers Dement. doi: 10.1016/j.jalz.2018.01.017. [Epub ahead of print].

Su, Y., Ryder, J., Li, B., Wu, X., Fox, N., Solenberg, P., et al. (2004). Lithium, a common drug for bipolar disorder treatment, regulates amyloid-beta precursor protein processing. Biochemistry 43, 6899-6908. doi: 10.1021/bi0 $35627 \mathrm{j}$

Subramanian, S., Bates, S. E., Wright, J. J., Espinoza-Delgado, I., and Piekarz, R. L. (2010). Clinical toxicities of histone deacetylase inhibitors. Pharmaceuticals 3 , 2751-2767. doi: 10.3390/ph3092751

Taher, N., Mckenzie, C., Garrett, R., Baker, M., Fox, N., and Isaacs, G. D. (2014). Amyloid-beta alters the DNA methylation status of cell-fate genes in an Alzheimer's disease model. J. Alzheimers Dis. 38, 831-844. doi: 10.3233/JAD131061

Tian, F., Marini, A. M, and Lipsky, R. H. (2010). Effects of histone deacetylase inhibitor Trichostatin A on epigenetic changes and transcriptional activation of Bdnf promoter 1 by rat hippocampal neurons. Ann. N.Y. Acad. Sci. 1199, 186-193. doi: 10.1111/j.1749-6632.2009.05175.X

Tohgi, H., Utsugisawa, K., Nagane, Y., Yoshimura, M., Genda, Y., and Ukitsu, M. (1999a). Reduction with age in methylcytosine in the promoter region -224 approximately -101 of the amyloid precursor protein gene in autopsy human cortex. Brain Res. Mol. Brain Res. 70, 288-292.

Tohgi, H., Utsugisawa, K., Nagane, Y., Yoshimura, M., Ukitsu, M., and Genda, Y. (1999b). The methylation status of cytosines in a tau gene promoter region alters with age to downregulate transcriptional activity in human cerebral cortex. Neurosci. Lett. 275, 89-92.

Tulloch, J., Leong, L., Thomson, Z., Chen, S., Lee, E. G., Keene, C. D., et al. (2018). Glia-specific APOE epigenetic changes in the Alzheimer's disease brain. Brain Res. 1698, 179-186. doi: 10.1016/j.brainres.2018.08.006

Wang, Z., Xu, P., Chen, B., Zhang Z, Zhang C, Zhan Q, et al. (2018). Identifying circRNA-associated-ceRNA networks in the hippocampus of Abeta1-42induced Alzheimer's disease-like rats using microarray analysis. Aging 10, 775-788. doi: 10.18632/aging.101427

Wu, P., Zuo, X., Deng, H., Liu, X., Liu, L., and Ji, A. (2013). Roles of long noncoding RNAs in brain development, functional diversification and neurodegenerative diseases. Brain Res. Bull. 97, 69-80. doi: 10.1016/j.brainresbull.2013. 06.001

Xie, B., Liu, Z., Liu, W., Jiang, L., Zhang, R., Cui, D., et al. (2017a). DNA Methylation and Tag SNPs of the BDNF gene in conversion of amnestic mild cognitive impairment into Alzheimer's disease: a cross-sectional cohort study. J. Alzheimers Dis. 58, 263-274. doi: 10.3233/JAD-170007

Xie, B., Xu, Y., Liu, Z., Liu, W., Jiang, L., Zhang, R., et al. (2017b). Elevation of peripheral BDNF promoter methylation predicts conversion from amnestic mild cognitive impairment to Alzheimer's disease: a 5-year longitudinal study. J. Alzheimers Dis. 56, 391-401. doi: 10.3233/JAD-160954 
Yang, B., Xia, Z. A., Zhong, B., Xiong X., Sheng C., Wang Y., et al. (2017). Distinct hippocampal expression profiles of long non-coding RNAs in an Alzheimer's disease model. Mol. Neurobiol. 54, 4833-4846. doi: 10.1007/s12035-0160038-5

Yang, T. T., Liu, C. G., and Gao, S. C. (2018). The serum exosome derived microRNA-135a, -193b, and -384 Were potential Alzheimer's disease biomarkers. Biomed. Environ. Sci. 31, 87-96. doi: 10.3967/bes2018.011

Zhang, C., Cheng, Y., Wang, H., Xu, J., Zhang, C., and Zhang, H. (2014). RNA interference-mediated knockdown of long-form phosphodiesterase-4D (PDE4D) enzyme reverses amyloid-beta42-induced memory deficits in mice. J. Alzheimers Dis. 38, 269-280. doi: 10.3233/JAD- 122236

Zhang, C. C., Xing, A., Tan, M. S., Tan, L., and Yu, J. T. (2016). The role of MAPT in neurodegenerative diseases: genetics, mechanisms and therapy. Mol. Neurobiol. 53, 4893-4904. doi: 10.1007/s12035-015-9415-8

Zhang, S., Zhu, D., Li, H., Li, H., Feng, C., and Zhang, W. (2017). Characterization of circRNA-associated-ceRNA networks in a senescence-accelerated mouse prone 8 brain. Mol. Ther. 25, 2053-2061. doi: 10.1016/j.ymthe.2017. 06.009

Zhang, T., Pang, P., Fang, Z., Guo Y, Li H, Li X., et al. (2017). Expression of BC1 impairs spatial learning and memory in Alzheimer's disease via APP translation. Mol. Neurobiol. 55, 6007-6020. doi: 10.1007/s12035-017-0820-z
Zhao, R., Hu, W., Tsai, J., Li, W., and Gan, W. B. (2017). Microglia limit the expansion of beta-amyloid plaques in a mouse model of Alzheimer's disease. Mol. Neurodegener. 12(1): 47. doi: 10.1186/s13024-017-0188-6

Zhu, H. C., Wang, L. M., Wang, M., Song, B., Tan, S., Teng, J. F., et al. (2012). MicroRNA-195 downregulates Alzheimer's disease amyloid-beta production by targeting BACE1. Brain Res. Bull. 88, 596-601. doi: 10.1016/j.brainresbull.2012. 05.018

Zovoilis, A., Agbemenyah, H. Y, Agis-Balboa, R. C., Stilling, R. M., Edbauer, D., Rao, P., et al. (2011). microRNA-34c is a novel target to treat dementias. EMBO J. 30, 4299-4308. doi: 10.1038/emboj.2011.327

Conflict of Interest Statement: The authors declare that the research was conducted in the absence of any commercial or financial relationships that could be construed as a potential conflict of interest.

Copyright (c) $2018 \mathrm{Liu}$, Jiao and Shen. This is an open-access article distributed under the terms of the Creative Commons Attribution License (CC BY). The use, distribution or reproduction in other forums is permitted, provided the original author(s) and the copyright owner(s) are credited and that the original publication in this journal is cited, in accordance with accepted academic practice. No use, distribution or reproduction is permitted which does not comply with these terms. 